\title{
Application of the normalized largest eigenvalue of structure tensor in the interpretation of potential field tensor data
}

\author{
Yuan Yuan ${ }^{1}$, Xiangyu Zhang ${ }^{2 *} \mathbb{B}$, Wenna Zhou ${ }^{3}$, Guochao Wu and Weidong Luo ${ }^{2}$
}

\begin{abstract}
Obtaining horizontal edges and the buried depths of geological bodies, using potential field tensor data directly is an outstanding question. The largest eigenvalue of the structure tensor is one of the commonly used edge detectors for delineating the horizontal edges without depth information of the potential field tensor data. In this study, we presented a normalized largest eigenvalue of structure tensor method based on the normalized downward continuation (NDC) to invert the source location parameters without any priori information. To improve the stability and accuracy of the NDC calculation, the Chebyshev-Pade' approximation downward continuation method was introduced to obtain the potential field data on different depth levels. The new approach was tested on various models data with and without noise, which validated that it can simultaneously obtain the horizontal edges and the buried depths of the geological bodies. The satisfactory results demonstrated that the normalized largest eigenvalue of structure tensor can describe the locations of geological sources and decrease the noise interference magnified by the downward continuation. Finally, the method was applied to the gravity data over the Humble salt dome in USA, and the nearbottom magnetic data over the Southwest Indian Ridge. The results show a good correspondence to the results of previous work.
\end{abstract}

Keywords: Potential field tensor data, Normalized largest eigenvalue, Structure tensor, Downward continuation, Source parameter estimation

\section{Introduction}

The source parameters estimation of potential field tensor data is an important part for geological interpretation, which can provide the horizontal position and the buried depth of the geological bodies as an a priori information for potential field inversion ( $\mathrm{Li}$ and Oldenburg 1998). The normalized full gradient (NFG) method proposed by the Russian Geophysical School (Elysseieva and Pašteka 2009, 2019) can be directly used to obtain

\footnotetext{
*Correspondence: zhang5821421@163.com

${ }^{2}$ MLR Key Laboratory of Marine Mineral Resources, Guangzhou Marine Geological Survey, Guangzhou 510075, China

Full list of author information is available at the end of the article
}

the depth of the causative sources by the normalization on the analytic signals modulus of the downward continuation potential field, without requiring any geometric input parameters or assumptions regarding geological properties.

In recent years, tremendous progresses have been made in the implementation of the NFG technique (Dondurur 2005; Aydin 2007; Oruç and Keskinsezer 2008; Fedi and Florio 2011; Pamukcu and Akcig 2011; Aghajani et al. 2011). Besides the potential field data, the NFG method has also been applied to the electromagnetic data (Dondurur 2005), self-potential data (Sindirgi et al. 2008) and seismic data (Karsli and Bayrak 2010). Fedi and Florio 
(2011) applied different normalization factors on analytical signals modulus and the downward continued potential field itself, and called this generalized method as normalized downward continuation (NDC). Based on the generalized NDC method, some authors have applied it on the local wavenumber (Ma et al. 2014), total horizontal derivatives ( $\mathrm{Li}$ et al. 2014), directional analytic signals (Zhou 2015) and directional total horizontal derivatives (Zhou et al. 2017) to obtain the horizontal edges and the buried depth simultaneously.

The structure tensor is one of the image processing techniques and presents a local orientation in an n-dimensional space (Weickert 1999a, b). The largest eigenvalues of the structure tensor of the potential field data have been used to delineate the edges of the geological bodies (Sertcelik and Kafadar 2012; Oruç et al. 2013; Yuan et al. 2014). In contrast to traditional derivativebased edge detection methods, the structure tensor has a property of which can reduce noise in the data while enhancing discontinuity boundaries, due to a Gaussian envelop within it. Based on the advantages of the largest eigenvalue of the structure tensor, in this paper, we apply the NDC on the largest eigenvalue of the structure tensor of the downward continued field to estimate the depth and the horizontal edges. In order to increase the stability of the NDC process, the Chebyshev-Pade' approximation downward continuation method developed by Zhou et al. (2018) was brought to compute each downward continuation level field. The synthetic models with and without noise and the real measured potential field data are utilized to validate the effectiveness of this new method.

\section{NDC of largest eigenvalue of structure tensor}

\section{Structure tensor of potential field tensor data}

Potential field gradient tensor data are the second-order space derivatives of potential field $f$ (gravitational field or magnetic field) in the three orthogonal directions $x, y$ and $z$. The potential field gradient tensor matrix is

$$
F=\left[\begin{array}{ccc}
f_{x x} & f_{x y} & f_{x z} \\
f_{y x} & f_{y y} & f_{y z} \\
f_{z x} & f_{z y} & f_{z z}
\end{array}\right]
$$

The original structure tensor consists of a Gaussian envelope and horizontal gradient tensor of the potential field data (Sertcelik and Kafadar, 2012). The expression of the original structure tensor matrix is where $G_{\delta}=\frac{1}{2 \pi \delta^{2}} e^{-\frac{1}{2}\left(\frac{x^{2}}{\delta x^{2}}+\frac{y^{2}}{\delta y^{2}}\right)}, \delta x$ and $\delta y$ are the standard deviations of Gaussian envelope in $x$ and $y$ directions. The homogeneous characteristic equation for $2 \mathrm{D}$ tensor $M$ is

$$
\lambda^{2}-\lambda\left(M_{11}+M_{22}\right)+\left(M_{11} M_{22}-M_{12} M_{21}\right)=0 .
$$

The largest eigenvalue of matrix $M$ is

$$
\lambda=\frac{1}{2}\left(M_{11}+M_{22}+\sqrt{\left(M_{11}-M_{22}\right)^{2}+4 M_{12} M_{21}}\right) .
$$

Sertcelik and Kafadar (2012) pointed that the largest eigenvalue $\lambda$ can locate the edges of geological bodies. However, the balancing ability of equalizing the edge signal amplitude of large and small anomalies is weak. The larger standard deviation $\delta x$ and $\delta y$ can enhance the balancing ability, but reduce the resolution of the identified edges.

\section{NDC of the largest eigenvalue}

The normalized full gradient (NFG) method (Elysseieva and Pasteka 2009; Zeng et al. 2002) is the normalization of the analytic signal modulus at different downward continuation levels. Fedi and Florio (2011) applied the normalization on the analytic signal and on the downward continuation potential field itself, called this generalized method as normalized downward continuation (NDC). The expression of NDC applied to the largest eigenvalue $\lambda$ of the structure tensor matrix can be expressed as:

$$
N \lambda=\frac{\lambda(x, y, z)}{N(z)}
$$

where $\lambda(x, y, z)$ is the edge detector of the structure tensor at point $(x, y, z) ; N(z)$ is the normalization function. Here, arithmetic mean, median and geometric mean are used as the normalization function, shown as:

$$
\left\{\begin{array}{l}
N(z)=\frac{1}{M} \sum_{0}^{M} \lambda(x, y, z) \quad \text { Arithmetic mean } \\
N(z)=\operatorname{median}(\lambda(x, y, z)) \quad \text { Median } \\
N(z)=\sqrt[M]{\lambda_{1} \lambda_{2} \cdots \lambda_{M}} \quad \text { Geometric mean }
\end{array}\right.
$$

where $M$ is the number of all calculation points.

$$
\mathrm{M}=G_{\sigma} *\left[\begin{array}{cc}
f_{z x}^{2} & f_{z x} f_{z y} \\
f_{z x} T_{z y} & f_{z y}^{2}
\end{array}\right]=\left[\begin{array}{cc}
G_{\sigma} * f_{z x}^{2} & G_{\sigma} * f_{z x} f_{z y} \\
G_{\sigma} * f_{z x} f_{z y} & G_{\sigma} * f_{z y}^{2}
\end{array}\right]=\left[\begin{array}{ll}
M_{11} & M_{12} \\
M_{21} & M_{22}
\end{array}\right],
$$


During the processing, there are many issues that needed to be overcome. The downward continuation plays the key role in the NDC method. The accuracy of the NDC method is determined by the stability of downward continuation method directly. Therefore, it is necessary to use a stable downward continuation in the calculation process. Many new stable algorithms have been introduced to implement the downward continuation method (Fedi and Florio, 2002; Cooper, 2004; Ma et al., 2013; Zeng et al., 2013, 2014; Zhang et al., 2013; Zhou et al., 2018). Zhou et al. (2018) has compared the errors between the function $\exp (x)$ and its different approximation functions, including Taylor series, Chebyshev approximation, Pade' approximation, and Chebyshev-Pade' approximation. Comparison of results indicate that downward continuation based on Chebyshev-Pade' approximation can obtain a more precise result. Therefore, in this study, the Chebyshev-Pade' approximation downward continuation method was chosen for the NDC of the largest eigenvalue of the structure tensor.

In the frequency domain, the downward continuation can be denoted as:

$$
\tilde{f}_{h}\left(\omega_{x}, \omega_{y}, \Delta h\right)=e^{\Delta h \omega_{\gamma}} \tilde{f}\left(\omega_{x}, \omega_{y}\right),
$$

where the downward continuation operator $e^{\Delta h \omega_{\gamma}}$ can be approximated by the (three and two) terms of Chebyshev-Pade' as follows (Zhou et al. 2018):

$$
e^{\Delta h \omega_{\gamma}}=\frac{0.9196+0.5667 \Delta h \omega_{\gamma}+0.1467\left(\Delta h \omega_{\gamma}\right)^{2}+0.01627\left(\Delta h \omega_{\gamma}\right)^{3}}{0.9194-0.3528\left(\Delta h \omega_{\gamma}\right)+0.0403\left(\Delta h \omega_{\gamma}\right)^{2}} .
$$

Therefore, the expression of the (three and two) terms of Chebyshev-Pade' approximation downward continuation is

$$
\tilde{f}_{h}\left(\omega_{x}, \omega_{y}, \Delta h\right)=\frac{0.9196+0.5667 \Delta h \omega_{\gamma}+0.1467\left(\Delta h \omega_{\gamma}\right)^{2}+0.01627\left(\Delta h \omega_{\gamma}\right)^{3}}{0.9194-0.3528\left(\Delta h \omega_{\gamma}\right)+0.0403\left(\Delta h \omega_{\gamma}\right)^{2}} \tilde{f}\left(\omega_{x}, \omega_{y}\right)
$$

where $f_{h}$ and $f$ are the gravity and magnetic data at two observation heights separated by a vertical distance $\Delta h$, respectively. $\tilde{f}_{h}$ and $\tilde{f}$ denotes the Fourier transform of $f_{h}$ and $f, \omega_{x}$ and $\omega_{y}$ are the wavenumbers $x$ - and $y$ - direction, and $\omega_{\gamma}=\sqrt{\omega_{x}^{2}+\omega_{y}^{2}}$ is the radial wavenumber. $\Delta h$ is a positive number for the downward continuation distance and vice versa for the upward continuation. Zhou et al. (2018) have pointed out that Chebyshev-Pade' approximation can give a more precise approximation in the low frequency and it can suppress the high frequency. During the calculation of downward continuation, the short wavelength (high frequency) components can be suppressed by Chebyshev-Pade' approximation. Therefore, we can obtain a stability and noise reduced potential field gradient data at different depths by using the Chebyshev-Pade' downward continuation. The residual noise of the potential field gradient data at different depths can be further removed by the Gaussian envelop when using the NDC of the largest eigenvalue of structure tensor to estimate the geology source.

Therefore, the calculation procedures of the NDC of

1. Calculate the potential field gradient data from the observed potential field data, or obtain from the real measurement.

2. Set a maximum depth $H$ (the depth that we want to know the maximum range of geologic source) for downward continuation, and divide the underground into $n$ layers $(i=1,2,3, \ldots, n)$.

3. alculate potential field gradient data at the different depth levels by using the Chebyshev-Pade' approximation downward continuation method. 
of the new proposed method. The thickness is $5 \mathrm{~m}$ and the contrasted density is $1 \mathrm{~g} / \mathrm{cm}^{3}$. The 3D view of the synthetic model and the synthetic gravity anomaly and gravity gradient components $f_{z x}$ and $f_{z y}$ are shown in Fig. 1. The Chebyshev-Pade' approximation downward continuation method is utilized to calculate different depth-level fields with the interval of $1 \mathrm{~m}$, and the maximum depth is set as $25 \mathrm{~m}$. In each depth level, arithmetic mean, median value and geometric mean are used as the normalization function to normalize the largest eigenvalue of the structure tensor. In order to compare the results conveniently, a cross section (the white line in Fig. 1b) is intercepted from the 3D result to display the character of the estimated depth results. Figure $2 \mathrm{a}-\mathrm{c}$ shows the cross-sectional results normalized by arithmetic mean, median and geometric mean with $\delta x=\delta y=0.01$. All of their maximum values (the white dot) can display the edge position and the center depth of the prism at $10 \mathrm{~m}$. Comparing these results, the maximum values normalized by the median display the center depth of the prism more clearly, shown in Fig. 2b. Figure $2 \mathrm{~d}$ shows the $3 \mathrm{D}$ view of the median normalized result at different depths. We can see that at the depth of $10 \mathrm{~m}$, the median normalized result can display the horizontal edge of the prism clearly. It demonstrates that we can obtain the source edge position and the buried depth information simultaneously by our new proposed method.

\section{Model 2: prism model with noise}

To capture the effect of noise in the new proposed method, we add $1 \%$ Gaussian noise to the synthetic gravity gradient anomalies in Fig. 1c, d. The noisy gravity gradients $f_{z x}$ and $f_{z y}$ are shown in Fig. 3. Figure 4 shows the cross section estimated depth results by the NDC of the largest eigenvalue of the structure tensor normalized by arithmetic mean, median and geometric mean with different standard deviations $\delta x$ and $\delta y$. We can see that all the results can display the depth information with the maximum values. As we have discussed in the theoretical part, the effect of noise can be suppressed by Chebyshev-Padé downward continuation. However, there are still some residual noise in the calculated potential field gradient data. The large standard deviation $\delta x$ and $\delta y$ can remove the residual noise disturbance more efficiently, and make the normalized results more clearly. However, with the increasing of the standard deviation $\delta x$ and $\delta y$, the resolution of the edge information of the prism have been reduced and may bring an error to the result. From Fig. 4i, when standard deviation $\delta x=10$ and $\delta y=10$, the estimated depth by geometric mean is $11 \mathrm{~m}$, with an error of $10 \%$. Therefore, a suitable standard deviation $\delta x$ and $\delta y$ can balance denoising ability and the resolution, and obtain a better result. However, the choice of a suitable standard deviation $\delta x$ and $\delta y$ depend on the noise level of the original measured potential data. A large noise level need choosing a large standard deviation, vice versa. In this model, we can see that Fig. 4 d, e with the standard deviation $\delta x=\delta \mathrm{y}=1$ get a better result than others, which can reduce the noise disturbance and retain the resolution of the identified horizontal edges.

Figure 5 displays the horizontal and vertical profiles of the distribution of the largest eigenvalues across, and along the edge of the model 1. From Fig. 5a, we can see that the normalized results by arithmetic mean, median and geometric mean all can demonstrate the horizontal edges model well. However, from Fig. 5b, we can see that the extent of the maximum values of the result normalized by median are corresponding to the vertical edges of the causative body. Therefore, we consider the normalized result by median can get a better result normalization factors arithmetic mean and geometric mean.

\section{Model 3: complex model with two prisms}

In order to test the application ability, a complex gravity model was constructed which contains two vertical-sided prisms with the center depths are $10 \mathrm{~km}$ and $15 \mathrm{~km}$. The thickness of both prisms is $5 \mathrm{~km}$. The contrasted density of the prisms is $1 \mathrm{~g} / \mathrm{cm}^{3}$ and $-2 \mathrm{~g} /$ $\mathrm{cm}^{3}$, respectively. The 3D view of the synthetic model and the synthetic gravity anomaly and gravity gradient anomalies $f_{z x}$ and $f_{z y}$ are shown in Fig. 6 . The downward continuation is utilized to calculate different depthlevel fields with an interval of $0.5 \mathrm{~km}$, and the maximum depth is set as $25 \mathrm{~km}$. Here, based on the results of model 1 and model 2, only the median was chosen as the normalization function to normalize the largest eigenvalue of the structure tensor in each depth level on this model. Figure 7c shows the cross section (white line in Fig. 6b) estimated depth results, with the maximum values of the normalized results by median are 10 and $15 \mathrm{~km}$ for prism 1 and prism 2, respectively. Figure $7 \mathrm{~d}$ shows the $3 \mathrm{D}$ view of the median value normalized result at different depths. We can see that at the depth of 10 and $15 \mathrm{~km}$, the median value normalized result can demonstrate the horizontal edge of the prism 

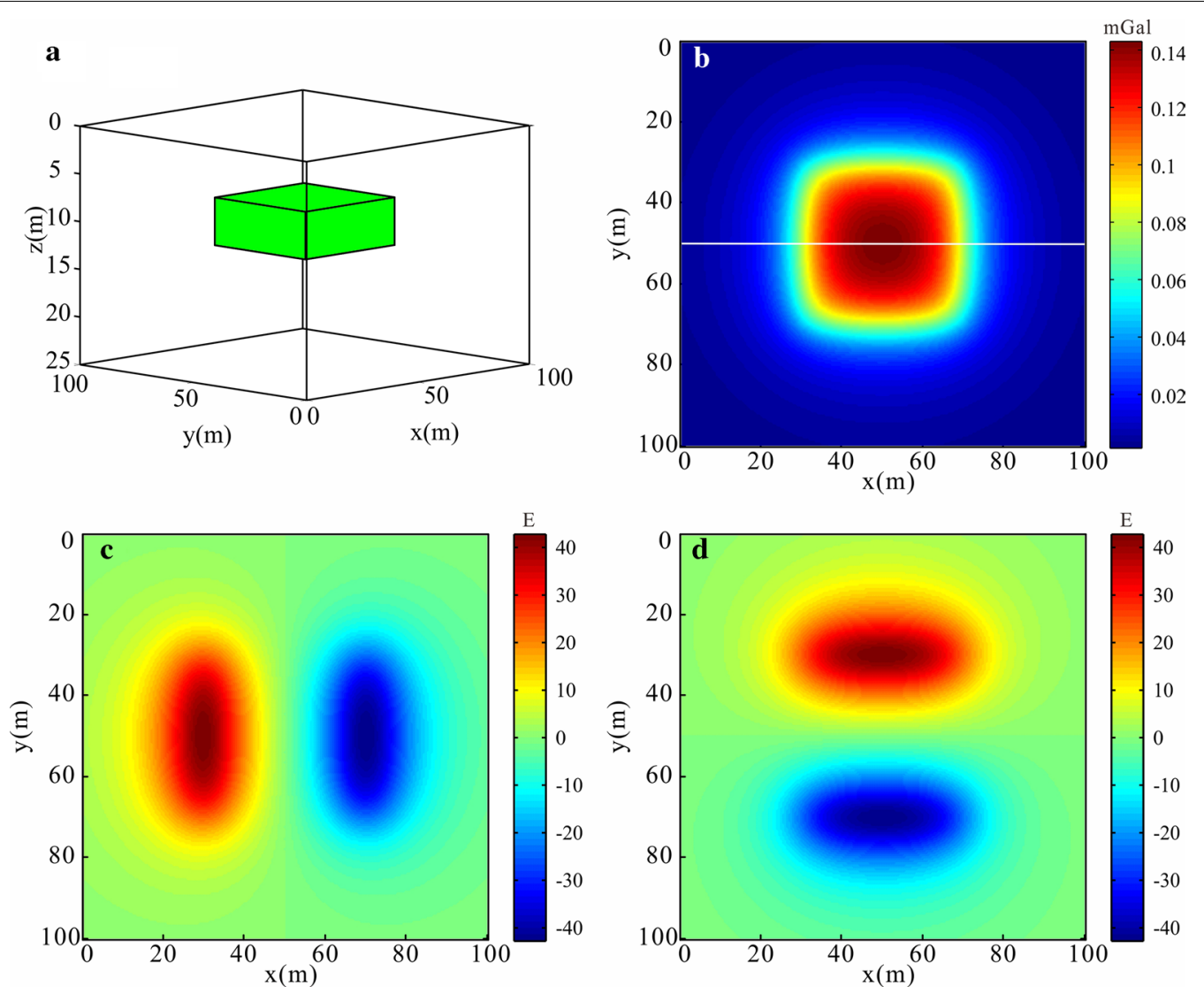

Fig. 1 Synthetic gravity anomaly and gravity gradient anomaly of a vertical-sided prism with the center depth of $10 \mathrm{~m}$. a 3D view of the model; $\mathbf{b}$ gravity anomaly, where the white line is the cross section for Fig. 2; c gravity gradient component $f_{z x}$; (d) gravity gradient component $f_{z y}$

1 and prism 2 clearly. Figure 7e displays the identified horizontal edges of the two prisms in plan view, which was extracted from Fig. $7 \mathrm{~d}$ by the method proposed by Blakley and Simpson (1986), which compared with the eight nearest neighbors of the identified horizontal edge grid in four directions (along the row, column, and both diagonals) to see if a maximum is present. We can see that the newly proposed method can obtain the source edge position and the buried depth information simultaneously.

We should reduce the magnetic anomaly to the pole before applying the new method to the magnetic anomaly to detect the horizontal edges and estimate the depth, as the horizontal derivatives of the magnetic anomaly are sensitive to magnetization direction (Ma and $\mathrm{Li} 2012$ ).

\section{Application to real potential field data} Gravity anomaly of Humble salt dome

To demonstrate the real application effect, the new method is applied to the residual Bouguer gravity anomaly caused by the Humble salt dome near Houston, Texas (Nettleton 1976), which was digitized by Shaw and Agarwal (Shaw and Agrawal 1997). Figure 8a shows the Bouguer anomaly where the grid interval is $100 \mathrm{~m}$. The horizontal gravity gradients $f_{z x}$ and $f_{z y}$ are calculated numerically from gravity data using the method proposed by Minkus and Hinojosa (2001), shown in Fig. 8b, c. The interval of the depth level is $0.3 \mathrm{~km}$, and the maximum depth is set as $9 \mathrm{~km}$. Figure 9 shows the depth estimated results of NDC of the largest eigenvalue of structure tensor by median. The estimated center depth of the salt dome is $4.2 \mathrm{~km}$ by the maximum value of the 

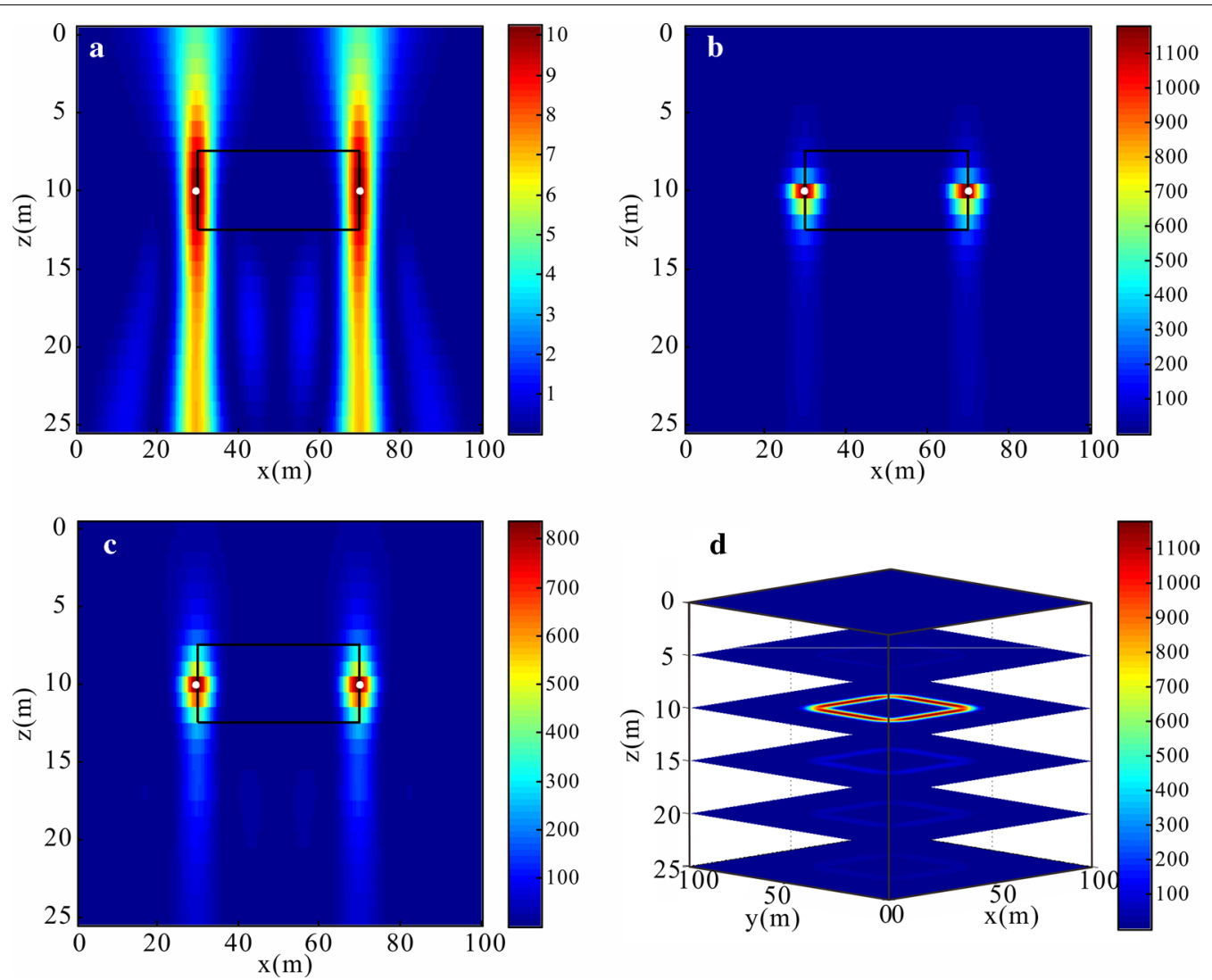

Fig. 2 Depth results estimated by the NDC of largest eigenvalue of structure tensor with different normalization functions, and the standard deviation in the Gaussian envelop is $\delta x=\delta y=0.01$. a By arithmetic mean; $\mathbf{b}$ by median; $\mathbf{c}$ by geometric mean; $\mathbf{d} 3 \mathrm{D}$ view of the normalized result by median
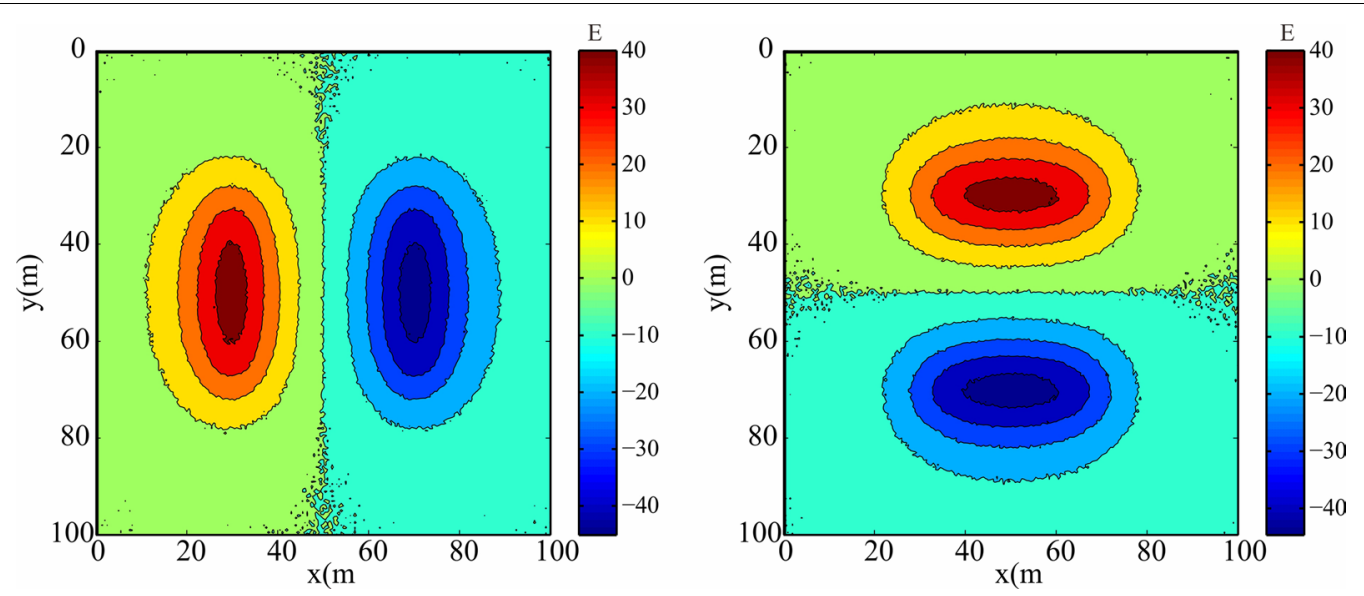

Fig. 3 Noisy gravity gradients of model 1 disturbed by $1 \%$ Gaussian noise. a Gravity gradient component $f_{z x}$; b gravity gradient component $f_{z y}$ 

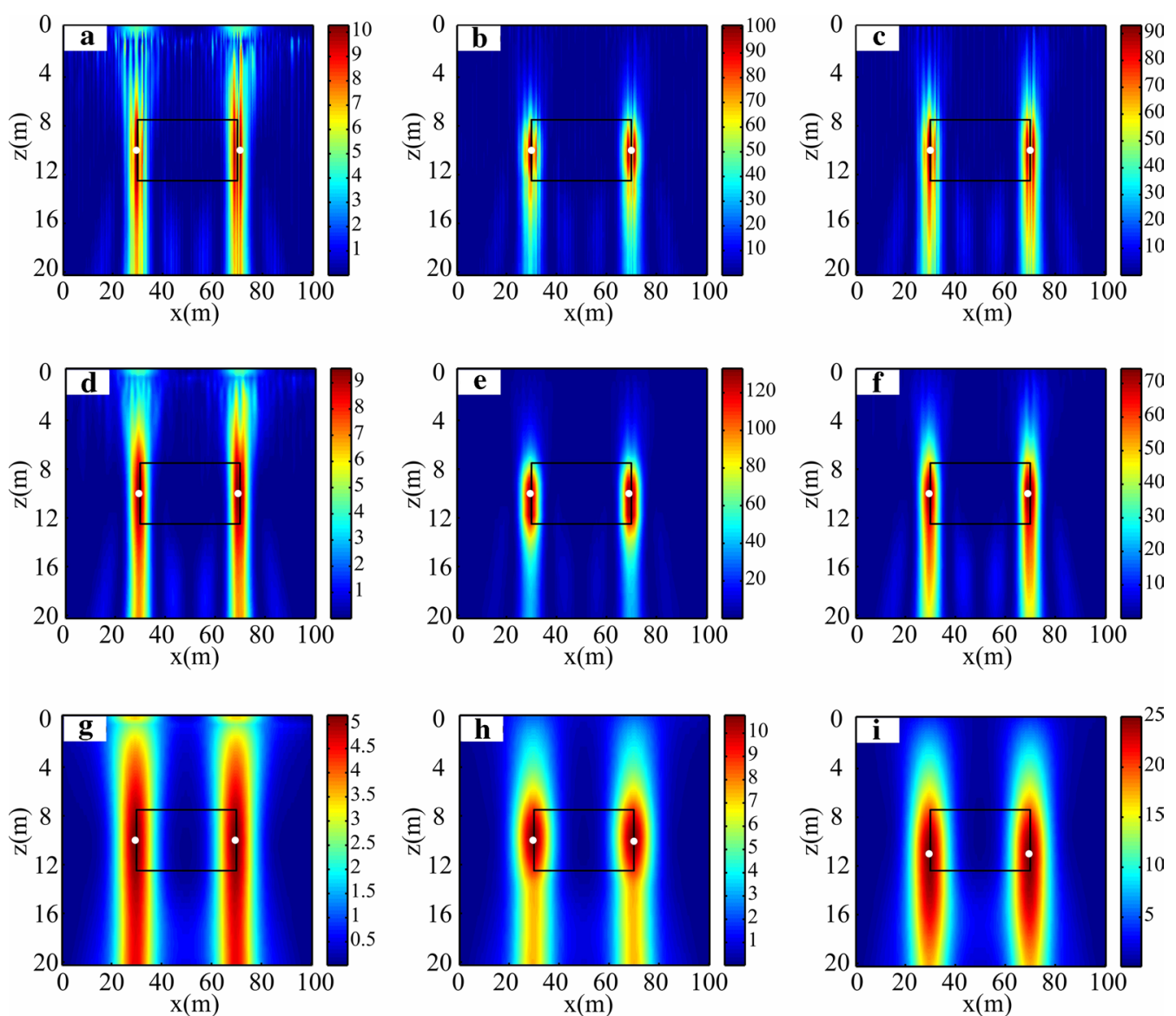

Fig. 4 Depth results estimated by the NDC of largest eigenvalue of structure tensor with different normalization functions and different standard deviations $\delta x$ and $\delta y$ in the Gaussian envelop. a By arithmetic mean $(\delta x=\delta y=0.1)$; b by median $(\delta x=\delta y=0.1)$; $\mathbf{c}$ by geometric mean $(\delta x=\delta \mathrm{y}=0.1) ; \mathbf{d}$ by arithmetic mean $(\delta x=\delta \mathrm{y}=1) ; \mathbf{e}$ by median $(\delta x=\delta \mathrm{y}=1) ; \mathbf{f}$ by geometric mean $(\delta x=\delta \mathrm{y}=1) ; \mathbf{g}$ by arithmetic mean $(\delta x=\delta y=10) ; \mathbf{h}$ by median $(\delta x=\delta y=10) ; \mathbf{i}$ by geometric mean $(\delta x=\delta y=10)$
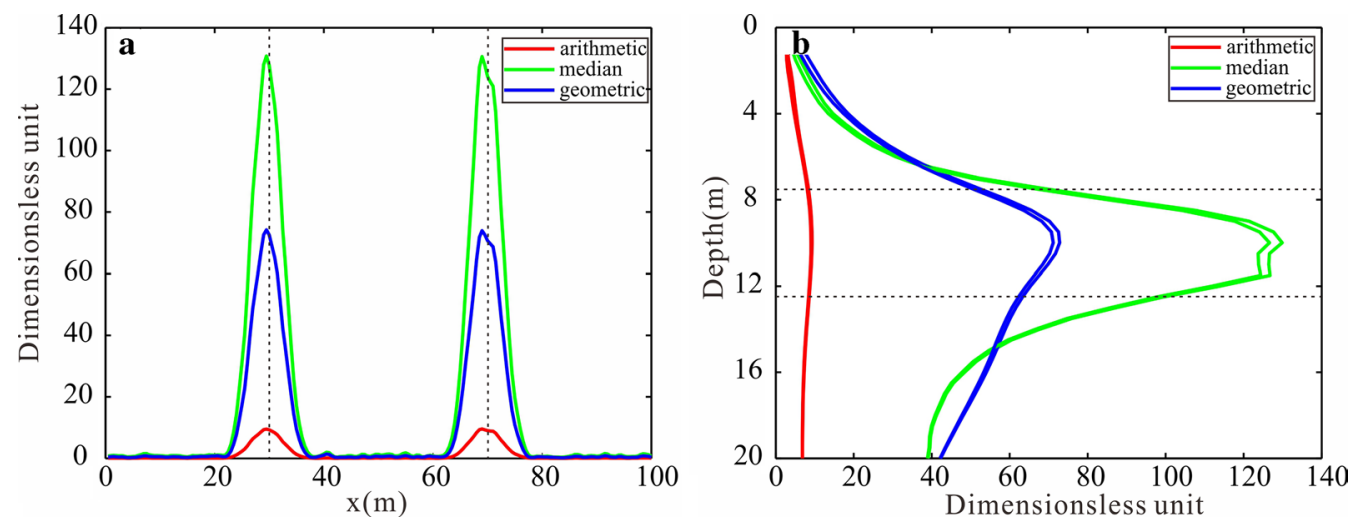

Fig. 5 The horizontal and vertical profiles of the distribution of the largest eigenvalues across, and along the edge of the model 1. (a) Horizontal profile; (b) vertical profile. The black dash lines outline the extent of the prism model in horizontal and vertical directions 
normalized results by median, shown in Fig. 9b. Besides, several other researchers have used different methods to interpret this anomaly. Table 1 is the comparison of the new method with previous salt dome depth estimation results, which proves the depth information obtained through the new method is reasonable. Figure 9c shows the horizontal slices of the NDC results normalized by the median at different depths, and Fig. 9d is the horizontal slice at the buried depth level $4.2 \mathrm{~km}$ in Fig. 9c, which indicates the horizontal extent of the dome in $\mathrm{E}-\mathrm{W}$ direction is about $4 \mathrm{~km}$ while in $\mathrm{N}-\mathrm{S}$ direction is about $2.5 \mathrm{~km}$.

\section{Near-bottom magnetic anomaly of Longqi hydrothermal} sulfides

Magnetic surveys have been established as an effective method to explore hydrothermal sulfides and study the internal structure of sulfide deposits (Tivey et al. 1993, 1996; Szitkar et al. 2014a, b; Szitkar and Dyment 2015). We use the new method to a near-bottom magnetic data over the first observed active high-temperature hydrothermal vent field at the ultraslow spreading Southwest Indian Ridge (SWIR). The data were collected by the autonomous underwater vehicle ABE (Autonomous Benthic Explorer) on board R/V DaYangYiHao with a fixed driving depth $2625 \mathrm{~m}$ in Feb.-Mar. 2007 (Zhu et al. 2010;
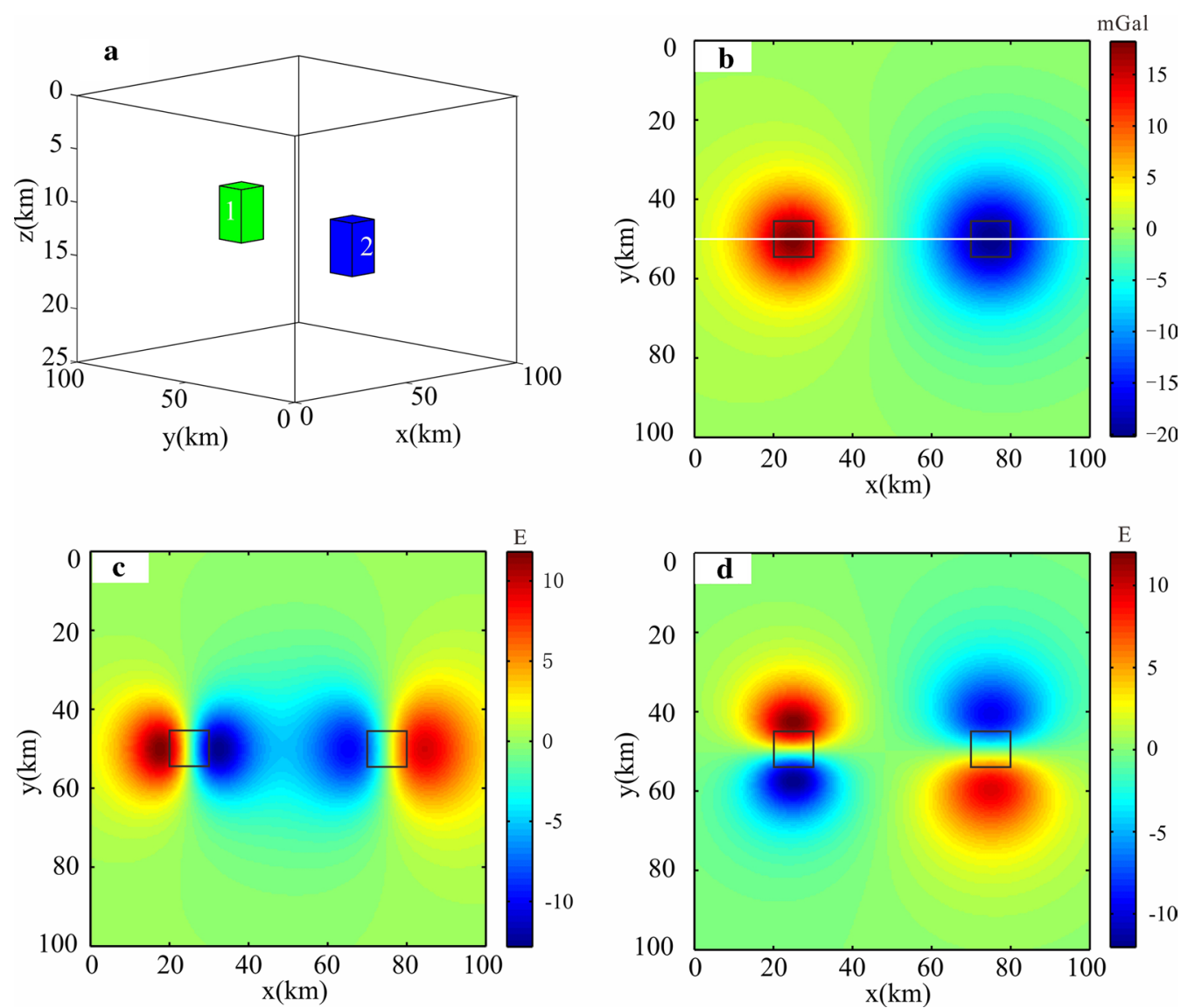

Fig. 6 Synthetic gravity anomaly and gravity gradient anomaly of a complex model contains two prisms with the center depths are $10 \mathrm{~km}$ and $15 \mathrm{~km}$, respectively. a $3 \mathrm{D}$ view of the model; b gravity anomaly; c gravity gradient component $f_{z x}$; d gravity gradient component $f_{z y}$. The black rectangle are the locations of the prisms in the horizontal plan, and the white line is the cross section for Fig. 7 

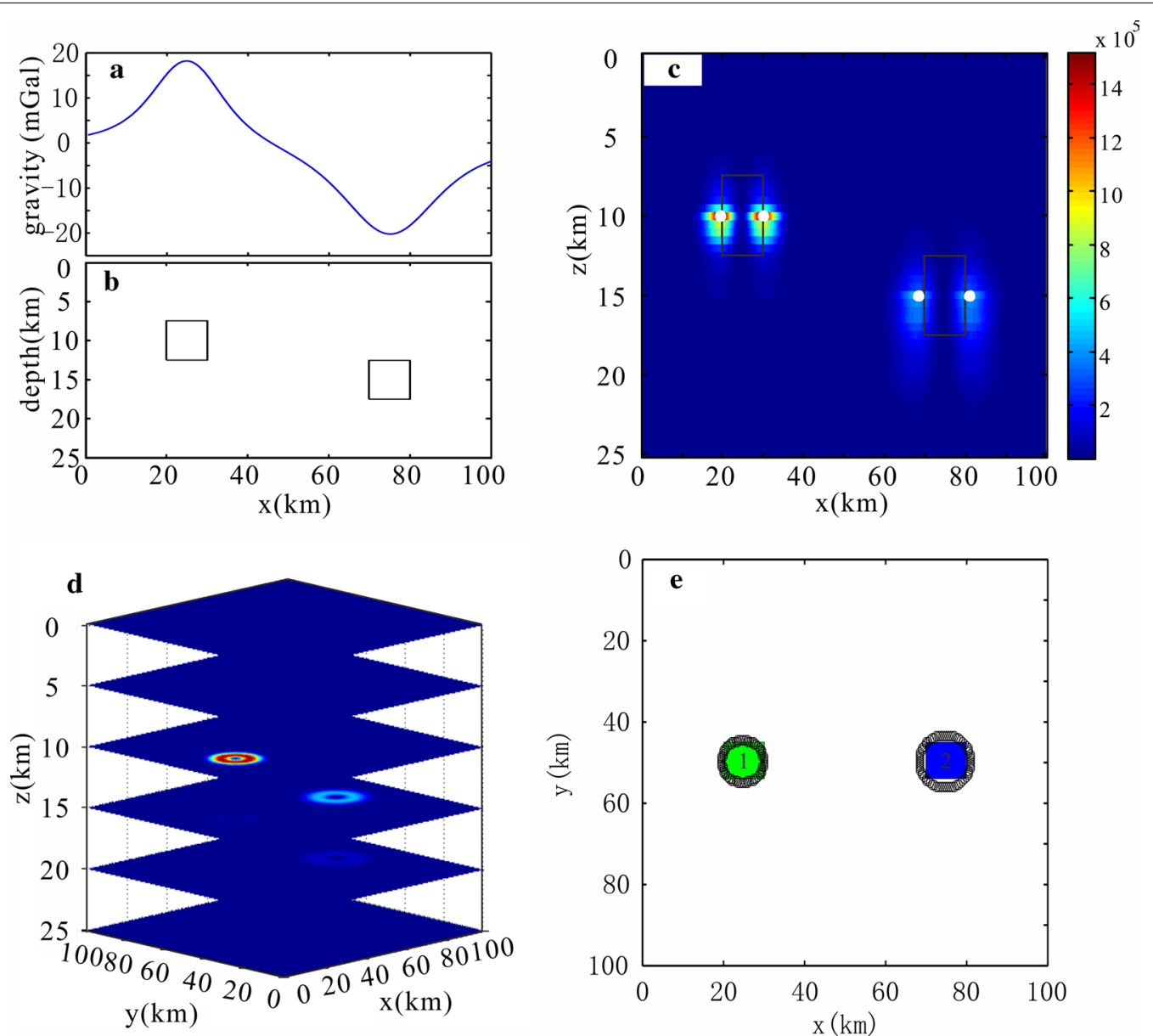

Fig. 7 Depth estimated of the NDC of largest eigenvalue of structure tensor by median normalization function. a Gravity anomaly of the cross section profile; $\mathbf{b}$ the positions of the two prisms in vertical cross section; $\mathbf{c}$ normalized depth result by median value; $\mathbf{d} 3 \mathrm{D}$ view of the normalized result by median value; e horizontal view of the identified edges of the prisms in Fig. 6a
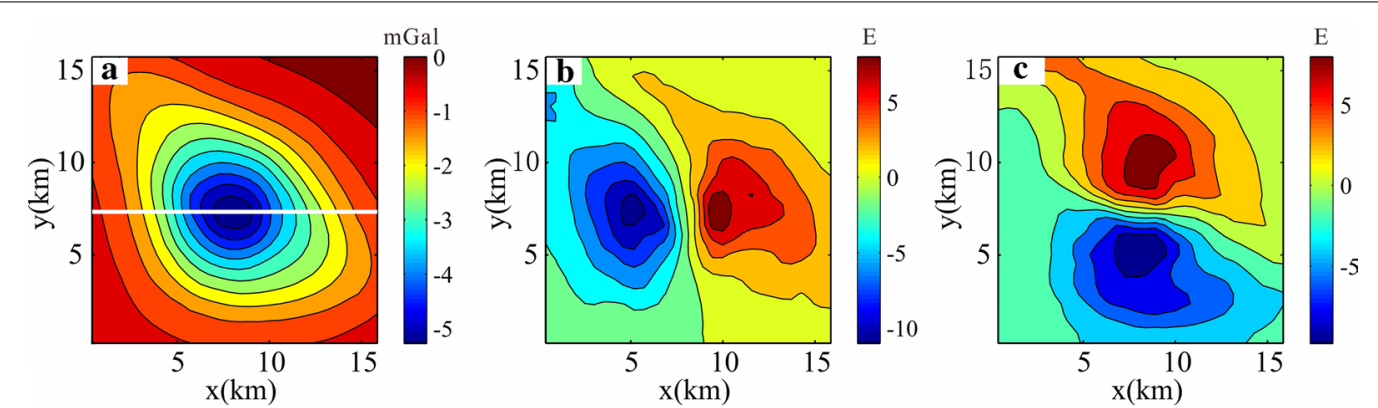

Fig. 8 Gravity anomaly caused by salt dome near Houston (Nettleton 1976) and the calculated tensor component. a Bouguer gravity anomaly; b gravity gradient component $f_{z x} ; \mathbf{c}$ gravity gradient component $f_{z y}$. The white line in Fig. $8 \mathrm{a}$ is the cross section for Fig. 9 

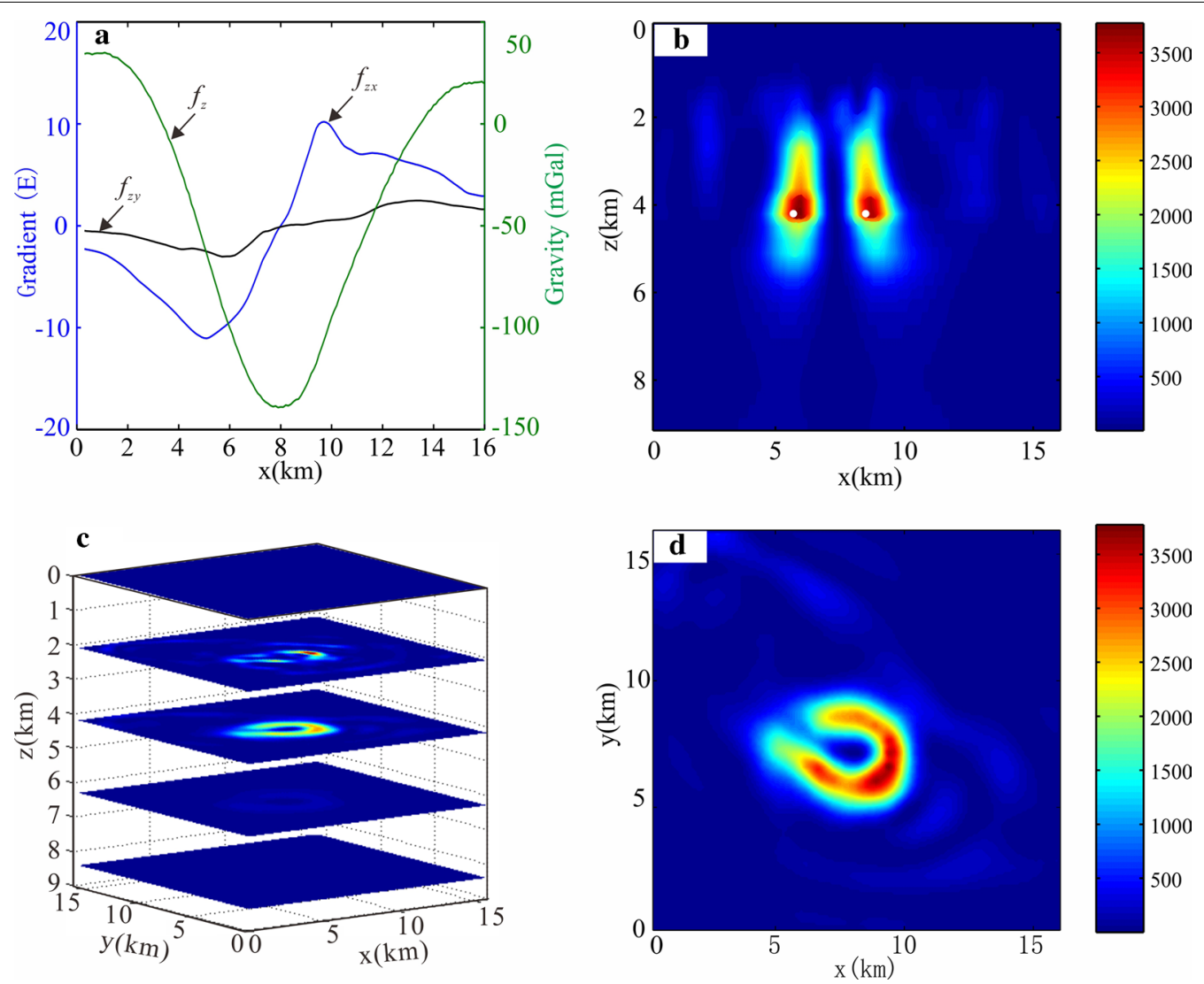

Fig. 9 Depth estimated of the NDC of largest eigenvalue of structure tensor by different normalization functions. a Gravity anomaly and gravity gradient anomaly of the cross section profile; $\mathbf{b}$ by median $(\delta x=\delta y=2) ; \mathbf{c}$ horizontal slices of 3D normalized result by median; $\mathbf{d}$ the slice at depth $4.2 \mathrm{~km}$ of $3 \mathrm{D}$ normalized result by median

Table 1 Center depth results of Humble salt dome from different methods

\begin{tabular}{ll}
\hline Method from different researches & Depth/km \\
\hline Shaw and Agarwal (1997) & 4.13 \\
Essa (2007) & 4.18 \\
Qruc (2010) & 4.12 \\
Ma et al. (2012) & 4.14 \\
Geng et al. (2016) & 4.5 \\
Zhou et al. (2017) & 4.25 \\
New method & 4.2 \\
New method & 4.20 \\
\hline
\end{tabular}

Tao et al. 2017). The measured line space is $250 \mathrm{~m}$. The measured magnetic anomaly reveals a strong correlation with the topography. Zhu et al. (2010) pointed out that the entire local topography high is an excess magnetic volume. Figure 10 displays the measured topography, magnetic anomaly and the calculated two magnetic gradient components $f_{z x}$ and $f_{z y}$. From Fig. 10a, the magnetic anomaly deviates from the local topography, which indicates that the root of this excess magnetic volume is tilt. Here, our new method was used to estimate the depth of the magmatic volume. In the downward continuation, the interval of the depth level is $0.05 \mathrm{~km}$, and the maximum depth is set as $2 \mathrm{~km}$. Figure 11 shows the depth estimated results of NDC of largest eigenvalue of structure tensor by the median value. We can see that the estimated center depth is between the 0.55 and $0.80 \mathrm{~km}$ by the maximum value of the normalized results by median below the measurement plane. When removing the depth of seawater, the estimated depth of the magmatic volume is between 0.404 and $0.583 \mathrm{~km}$ below the 


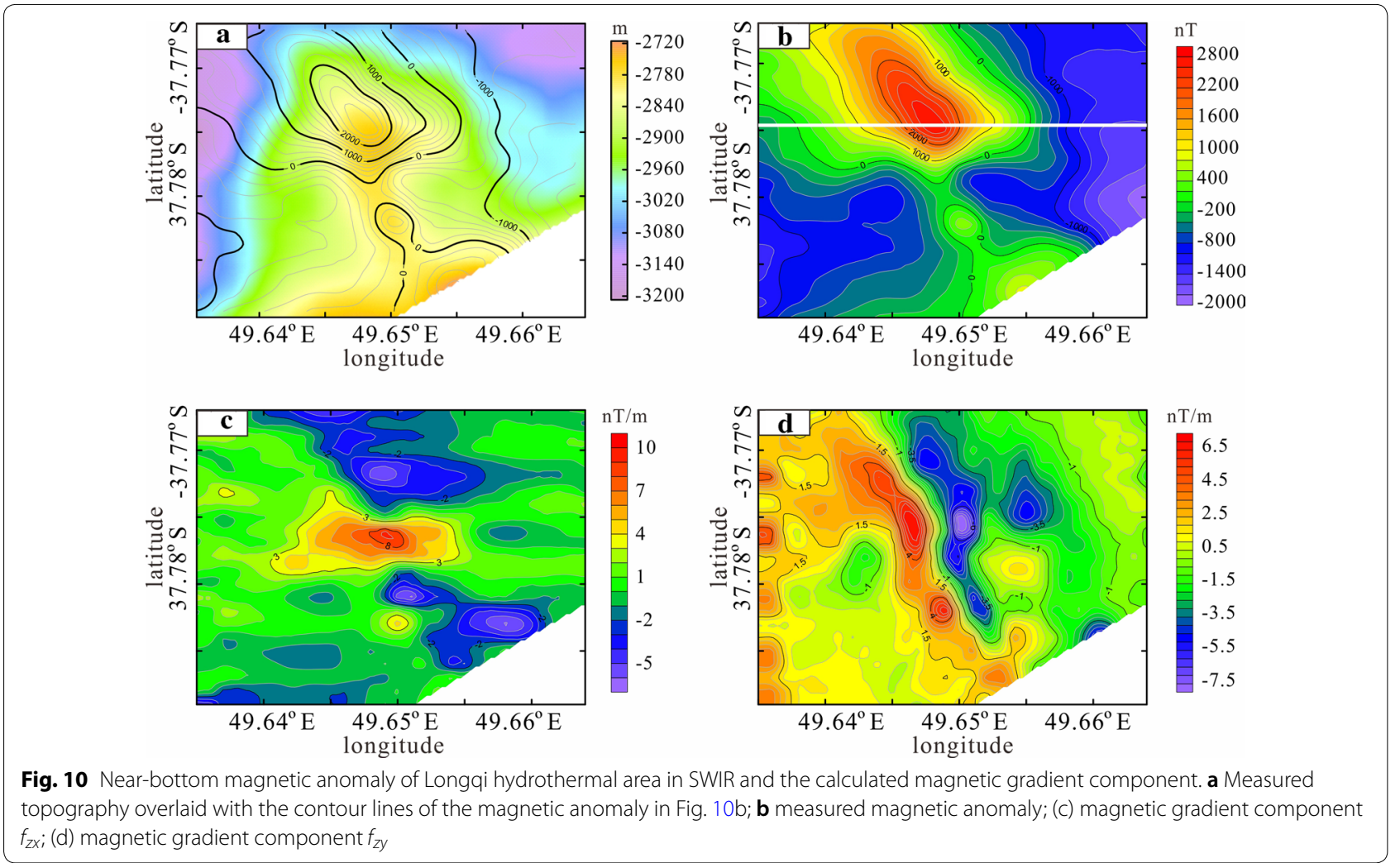

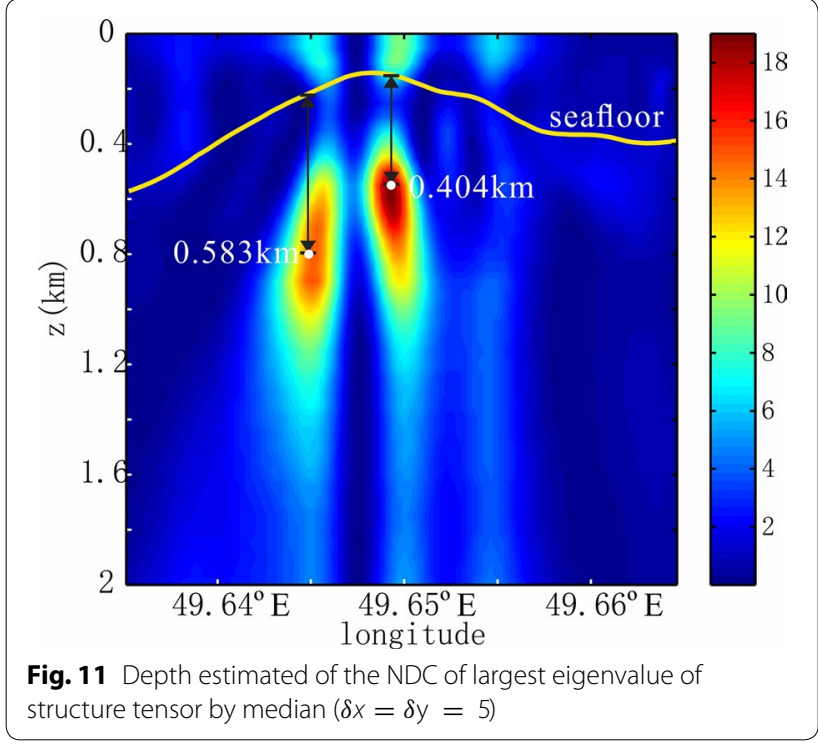

seafloor. The estimated depth shows that magmatic volume is tilted, consistent with the interpretation result in Fig. 10a.

\section{Conclusions}

We have presented a source parameters estimation method based on the NDC of the largest eigenvalue of the structure tensor of the potential field tensor data, which can simultaneously locate the horizontal edges and the buried depth of the geological sources. The maximum value in 3D space can locate the position. Due to the use of a Gaussian envelop filter in the structure tensor, the new proposed method can reduce the noise effect that is magnified by the downward continuation. The method has been tested by synthetic data with and without noise, and satisfactory results are obtained. Finally, the method was applied to the real measured gravity data over the Humble salt dome in USA and the near-bottom magnetic data over the Southwest Indian Ridge successfully. The results show a good correspondence to the previous work results.

\section{Abbreviations}

NDC: Normalized downward continuation; NFG: Normalized full gradient; SWIR: Southwest Indian Ridge; ABE: Autonomous Benthic Explorer. 


\section{Acknowledgements}

We express our sincere gratitude to the editors and reviewers in improving the manuscript, and acknowledge the captain and crews of the DY115-19 cruises on R/V DayangYihao, the Autonomous Benthic Explorer (ABE) group, who contributed to the success of measuring the magnetic anomaly in SWIR.

\section{Authors' contributions}

In this study, $Y Y$ and $X Z$ conceived and designed the method. $Y Y, X Z$ and $W Z$ were responsible for the model data and real data analysis. $Y Y$ wrote the main manuscript text. All authors read and approved the final manuscript.

\section{Funding}

This study is supported by the Fundamental Research Funds for National Natural Science Foundation of China $(41804169,41906197)$, Project of China Geological Survey (DD20191002), COMRA Major Project under contract (DY135-S1-01-06), Basic Scientific Research Special Fund Project of Second Institute of Oceanography, MNR (JP1902), National key research and development plan (SQ2018YFF020145), Natural Science Foundation of Zhejiang Province, China (LQ18D040001).

\section{Availability of data and materials}

The datasets used and/or analyzed during the current study are available from the corresponding author on any request.

\section{Competing interests}

The authors declare that they have no competing interests.

\section{Author details}

${ }^{1}$ Key Laboratory of Submarine Geosciences, Second Institute of Oceanography, Ministry of Natural Resources, Hangzhou 310012, China. ${ }^{2}$ MLR Key Laboratory of Marine Mineral Resources, Guangzhou Marine Geological Survey, Guangzhou 510075, China. ${ }^{3}$ School of Earth Sciences, Key Laboratory of Mineral Resources in Western China (Gansu Province), Lanzhou University, Lanzhou 730000, Gansu, China.

\section{Received: 10 August 2020 Accepted: 26 September 2020}

Published online: 08 October 2020

\section{References}

Aghajani H, Moradzadeh A, Zeng H (2011) Detection of high-potential oil and gas fields using normalized full gradient of gravity anomalies: a case study in the Tabas Basin, Eastern Iran. Pure Appl Geophys 168:1851-1863

Aydin A (2007) Interpretation of gravity anomalies with the normalized full gradient (NFG) method and an example. Pure Appl Geophys 164:2329-2344

Blakley RJ, Simpson RW (1986) Approximating edges of source bodies from magnetic and gravity anomalies. Geophysics 51:1494-1490

Cooper GRJ (2004) The stable downward continuation of potential field data. Explor Geophys 35:260-265

Dondurur D (2005) Depth estimates for Slingram electromagnetic anomalies from dipping sheet-like bodies by the normalized full gradient method. Pure Appl Geophys 162:2179-2195

Elysseieva IS, Pasteka R (2009) Direct interpretation of 2D potential fields for deep structures by means of the quasi-singular points method. Geophys Prospect 57:683-705

Elysseieva IS, Pasteka R (2019) Historical development of the total normalized gradient method in profile gravity field interpretation. Geophys prospect 67:188-209

Essa KS (2007) Gravity data interpretation using the s-curves method. J Geophys Eng 4(2):204-213

Fedi M, Florio G (2002) A stable downward continuation by using ISVD method. Geophys J Int 151:146-156

Fedi M, Florio G (2011) Normalized downward continuation of potential fields within the quasi-harmonic region. Geophys Prospect 59:1087-1100

Geng MX, Huang DN, Yu P, Yang Q (2016) Three-dimensional constrained inversion of full tensor gradiometer data based on cokriging method. Chinese J Geophys 59(5):1849-1860
Karsli H, Bayrak Y (2010) Application of the normalized total gradient (NTG) method to calculate envelope of seismic reflection signals. J Appl Geophys 71:90-97

Li LL, Huang DN, Han LG (2014) Application of the normalized total horizontal derivative (NTHD) in the interpretation of potential field data. Chinese J Geophys 57(12):4123-4131

Li Y, Oldenburg DW (1998) 3D inversion of gravity data. Geophysics 63:109-119

Ma GQ, Du XJ, Li LL (2012) Comparison of the tensor local wavenumber method with the conventional local wavenumber method for interpretation of total tensor data of potential fields. Chinese J Geophys 55(7):2450-2461

Ma GQ, Huang DN, Li LL, Yu P (2014) A normalized local wavenumber method for interpretation of gravity and magnetic anomalies. Chinese J Geophys 57(4):1300-1309

Ma GQ, Li LL (2012) Edge detection in potential fields with the normalized total horizontal derivative. Comput Geosci 41:83-87

Ma GQ, Liu C, Huang DN, Li LL (2013) A stable iterative downward continuation of potential field data. J Appl Geophys 98:205-211

Minkus KL, Hinojosa JH (2001) The complete gravity gradient tensor derived from the vertical component of gravity: a Fourier transform technique. J Appl Geophys 46:159-174

Nettleton LL (1976) Gravity and magnetics in oil prospecting. McGraw-Hill Book Co., New York, pp 460-468

Oruç B (2010) Depth estimation of simple causative sources from gravity gradient tensor invariants and vertical component. Pure Appl Geophys 167(10):1259-1272

Oruç B, Keskinsezer A (2008) Detection of causative bodies by normalized full gradient of aeromagnetic anomalies from east Marmara region, NW Turkey. J Appl Geophys 65:39-49

Oruç B, Sertçelik I, Kafadar Ö, Selim HH (2013) Structural interpretation of the Erzurum Basin, eastern Turkey, using curvature gravity gradient tensor and gravity inversion of basement relief. J Appl Geophys 88:105-113

Pamukcu OA, Akcig Z (2011) Isostasy of the Eastern Anatolia (Turkey) and discontinuities of its crust. Pure Appl Geophys 168:901-917

Sindirgi P, Pamukcu O, Ozyalin S (2008) Application of normalized full gradient method to self potential (SP) data. Pure Appl Geophys 165:409-427

Sertcelik I, Kafadar O (2012) Application of edge detection to potential field data using eigenvalue analysis of structure tensor. J Appl Geophys 84:127-136

Shaw RK, Agarwal BNP (1997) A generalized concept of resultant gradient to interpret potential field maps. Geophys Prospect 45(3):513-520

Szitkar F, Dyment J, Choi Y, Fouquet Y (2014a) What causes low magnetization at basalt-hosted hydrothermal sites? Insights from inactive site Krasnov (MAR 16380 N). Geochem Geophy Geosy 15(4):1441-1451

Szitkar F, Dyment J, Fouquet Y, Honsho C, Horen H (2014b) The magnetic signature of ultramafic-hosted hydrothermal sites. Geology 42(8):715-718

Szitkar F, Dyment J (2015) Near-seafloor magnetics reveal tectonic rotation and deep structure at the TAG (Trans-Atlantic Geotraverse) hydrothermal site (Mid-Atlantic Ridge, 26 N). Geology 43(1):87-90

Tao C, Wu T, Liu C, Li H, Zhang J (2017) Fault inference and boundary recognition based on near-bottom magnetic data in Longqi hydrothermal field. Mar Geophys Res 38:17-25

Tivey MA, Rona PA, Kleinrock MC (1996) Reduced crustal magnetization beneath relict hydrothermal mounds: TAG hydrothermal field, MidAtlantic Ridge, 26 N. Geophys Res Lett 23(23):3511-3514

Tivey MA, Rona PA, Schouten H (1993) Reduced crustal magnetization beneath the active sulfide mound, TAG hydrothermal field, Mid-Atlantic Ridge at 26 N. Earth planet sc lett 115(1-4):101-115

Weickert J (1999a) Coherence-enhancing diffusion of color images. Image Vision Comput 17:199-210

Weickert J (1999b) Coherence-enhancing diffusion filtering. Int J Comput Vision 31:111-127

Yuan Y, Huang DN, Yu OL, Lu PY (2014) Edge detection of potential field data with improved structure tensor methods. J Appl Geophys 108:35-42

Zeng HL, Meng XH, Yao CL, Li XM, Lou H, Guang ZN, Li ZP (2002) Detection of reservoirs from normalized full gradient of gravity anomalies and its application to Shengli oil field, east China. Geophysics 67:1138-1147 
Zeng XN, Li XH, Su J, Liu DZ, Zou HX (2013) An adaptive iterative method for downward continuation of potential-field data from a horizontal plane. Geophysics 78:J43-J52

Zeng XN, Liu DZ, Li XH, Chen DX, Niu C (2014) An improved regularized downward continuation of potential field data. J Appl Geophys 106:114-118

Zhang HL, Ravat D, Hu XY (2013) An improved and stable downward continuation of potential field data: The truncated Taylor series iterative downward continuation method. Geophysics 78:J75-J86

Zhou S, Jiang JB, Lu PY (2017) Source parameters estimation by the normalized downward continuation of gravity gradient data. J Appl Geophys 147:81-90

Zhou W, Li J, Yuan Y (2018) Downward continuation of potential field data based on Chebyshev-Pade' approximation function. Pure Appl Geophys 175:275-286
Zhou WN (2015) Normalized full gradient of full tensor gravity gradient based on adaptive iterative Tikhonov regularization downward continuation. J Appl Geophys 118:75-83

Zhu J, Lin J, Chen YJ, Tao C, German CR, Yoerger DR, Tivey MA (2010) A reduced crustal magnetization zone near the first observed active hydrothermal vent field on the Southwest Indian Ridge. Geophys Res Lett 37(18):L18303

\section{Publisher's Note}

Springer Nature remains neutral with regard to jurisdictional claims in published maps and institutional affiliations.

\section{Submit your manuscript to a SpringerOpen ${ }^{\circ}$ journal and benefit from:}

- Convenient online submission

- Rigorous peer review

- Open access: articles freely available online

- High visibility within the field

- Retaining the copyright to your article

Submit your next manuscript at $\boldsymbol{\nabla}$ springeropen.com 